\title{
Correlative Tomography - Bridging the length-scales through correlative X-ray and Electron Imaging
}

Philip Withers ${ }^{1}$, Jack Donoghue ${ }^{1}$ and Timothy Burnett ${ }^{2}$

${ }^{1}$ University of Manchester, Manchester, England, United Kingdom, ${ }^{2}$ The University of Manchester, Manchester, United Kingdom

Nearly all materials, whether naturally occurring or manufactured, have a range of length scales that must be considered in terms of understanding their behaviour. At the same time there is no technique that can image a materials microstructure across all the relevant scales (Figure 1). In the past a multiscale picture has usually be constructed from the analysis of different samples observed (usually in two dimensions (2D)) using different instruments and the information either combined either qualitatively or statistically to establish processing - microstructure - property relationships.

Recent advances in X-ray computed tomography (CT) has significantly advanced the level of information that can be obtained non-destructively about materials structures at scales from the meso ( $>10 \mathrm{~mm})$, micro (down to sub-micron) and nano scales (tens of $\mathrm{nm}$ ). Alongside this, advances in serial sectioning tomography using scanning electron microscopes (SEM) combined with focused ion, plasma ion beam or pulsed laser milling has extended the level of (microstructural, crystallographic or chemical) information that can be obtained destructively in 3D over volumes from 50micron, to 200micron to $1 \mathrm{~mm}$ volumes respectively [1].

In this paper we look at how correlative tomography which brings together volume imaging by X-ray and electron can provide both multiscale and multi-model information. Through a series of examples we will demonstrate various applications of correlative 3D imaging, namely correlative tomography. In addition we will illustrate the potential of emerging triple beam microscopes (bringing together laser- plasma focused ion and electron beams) can play a pivotal role lining X-ray and electron microscopy within correlative workflows. We will look at how X-ray CT is invaluable in monitoring structural evolution over time, for example materials degradation or damage accumulation, and how this can be complemented by region of interest electron tomography to provide key insights at a finer level, augmented by crystallographic and chemical information. Taken together the behaviour across the scales can provide key information about potential routes to slow degradation processes such as corrosion, wear and cracking. In addition we will show how X-ray CT can be used to experimentally steer in-depth 3D electron microscopical studies. Through these examples we will discuss both the practical issues relating to correlative workflows but also the issues and opportunities relating to the co-visualisation and analysis of correlative datasets.

\section{Acknowledgements}

The authors are grateful to the EPSRC for access to facilities in the Multidisciplinary Characterisation Facility (MCF) within the Henry Royce Institute (EP/P025021/1). In particular we acknowledge the support for the Henry Moseley X-ray Imaging Facility through grants (EP/F007906/1, EP/F001452/1, EP/I02249X, EP/M010619/1, EP/F028431/1, EP/M022498/1 and EP/T02593X/1). PJW acknowledges support from the European Research Council grant No. 695638 CORREL-CT. We are also grateful to Thermo Fisher and Zeiss for their support in the development of correlative 3D imaging. 


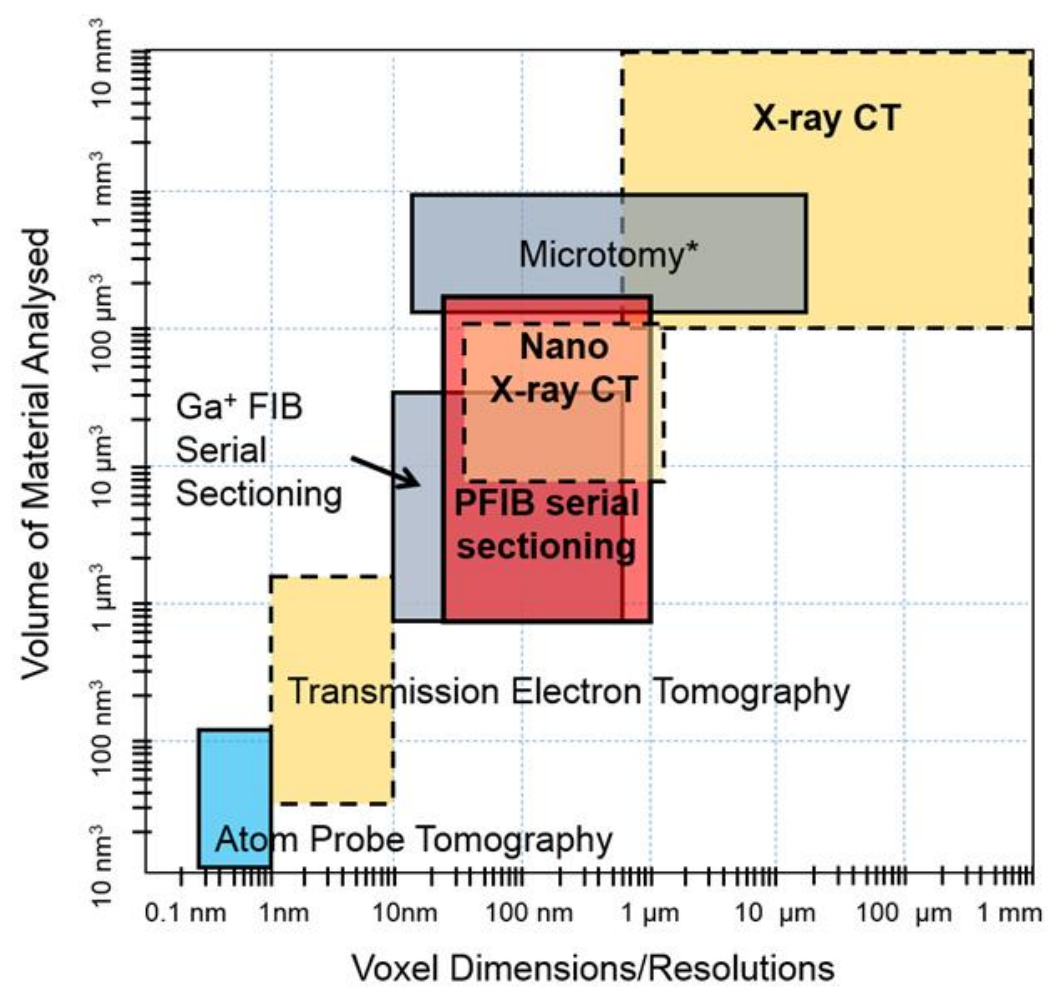

Figure 1. Figure 1: In order to capture information over a range of scales electron and X-ray imaging techniques must be brought together correlatively [2].

\section{References}

1. Echlin, M.P., et al., Serial sectioning in the SEM for three dimensional materials science. Current Opinion in Solid State and Materials Science, 2020. 24(2): p. 100817.

2. Withers, P.J. and T.L. Burnett, Rich multi-dimensional correlative imaging. IOP Conference Series: Materials Science and Engineering, 2019. 580: p. 012014. 